\title{
La vida de Dios en Zubiri. Estudio desde el curso inédito El problema de Dios (1948-1949)*
}

\author{
Enzo Solari - Esteban Vargas \\ PONTIFICIA UNIVERSIDAD CATÓLICA DE VALPARAÍSO \\ enzo.solari@ucv.cl - esteban.vargas@ucv.cl
}

\begin{abstract}
Resumen: Este texto presenta la idea de vida que sostiene Zubiri, indaga qué es la vida de Dios según el mismo y, en fin, reconstruye cuál es la teoría del significado de los enunciados teológico-filosóficos que emergen de las ideas maduras de Zubiri, considerando también otras ideas filosóficas tradicionales y contemporáneas acerca de la vida divina.
\end{abstract}

Palabras clave: Vida, Dios, enunciado, analogía, univocidad, metáfora, fenomenología

Abstract: By looking at Zubiri's idea of life, this paper seeks to look into what divine life is according to him so as to propound a theory of the meaning of theological and philosophical statements in the mature work of the Spanish philosopher while weighing other traditional and contemporaneous ideas about divine life.

Keywords: Life, God, statement, analogy, univocity, metaphor, phenomenology.

Tal como es tradicional hablar de Dios negativa o positivamente, literal o analógicamente, metafórica o aun paradójicamente, así también lo es aplicar a la divinidad el atributo de la vida.

Este texto se ha beneficiado del financiamiento otorgado por becas C de Stipendienwerk Lateinamerika-Deutschland (Icala, Consejo Valparaíso, Chile) a dos proyectos de investigación: El ateísmo en el curso El problema de Dios de Xavier Zubiri, y Dios y el origen del hombre a la luz del curso El problema de Dios (1948-1949) de Xavier Zubiri (2012-2013), a cargo de los profesores Enzo Solari y Esteban Vargas respectivamente. También ha sido apoyado por la Fundación Xavier Zubiri de Madrid, que permitió el acceso a sus archivos inéditos. 
Esto último ya se decía antiguamente. Por ej. Aristóteles: “y en él [en Dios] surge vida, pues la actividad de la inteligencia es vida y él se identifica con tal actividad. Y su actividad es, en sí misma, vida óptima y eterna. Afirmamos, pues, que Dios es un viviente eterno y óptimo. Así pues, a Dios corresponde vivir una vida continua y eterna. Esto es,

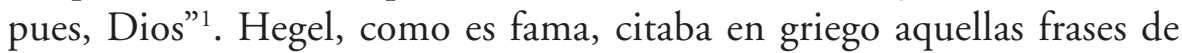
Aristóteles justo al terminar su Enzyklopädie. Antes Kant, en su Opus postumum, mientras se las había con Spinoza, el panteísmo y su propio teísmo moral, todo a la luz del ajuste incansable de su propia filosofía transcendental, no dejaba de hablar de Dios como viviente: "bajo el concepto de Dios se piensa una sustancia con conciencia adecuada a todos los fines, es decir una persona en la que la expresión, reforzada tautológicamente, de Dios vivo sirve tan solo para designar la personalidad de tal ser: ser omnipotente (ens summum), omnisapiente (summa inteligentia) e infinitamente bueno (summum bonum)" 2 .

Zubiri no escapa a esta tradición. Su propia postura al respecto, en cualquier caso, no es fácilmente interpretable. Además, tenemos el problema de que los comentarios usuales a la filosofía de Zubiri suelen despegarse muy magramente de los propios giros zubirianos, con lo que dicha literatura secundaria tiende a volverse repetitiva y contentarse con el parafraseo. Falta, pues, un enfrentamiento más especulativo con esa postura de Zubiri según la cual cabe predicar la vida de Dios. Tal es la pretensión de estas páginas: abordar especulativamente qué significa vida en Zubiri y, sobre todo, qué es la vida cuyo modo de realidad es no pura relatividad, sino absolutidad, y una reduplicativa y plena.

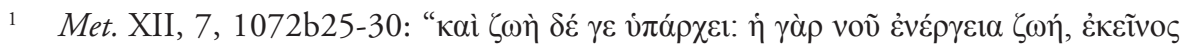

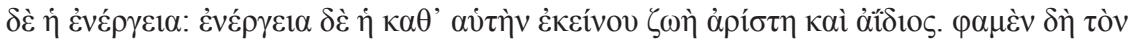

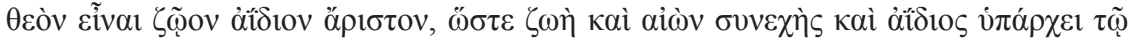

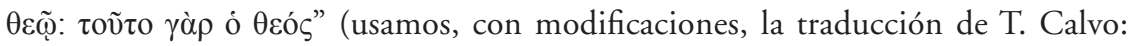
Metafísica, Gredos, Madrid, 1994, p. 488).

2 Opus postumum, en Akademie Ausgabe Vol. XXII, p. 48 (texto fechable en el otoño de 1800-1801): "unter dem Begriffe von Gott denkt man sich eine Substanz die allen Zwecken mit Bewustseyn angemessen d.i. eine Person wobey der tavtologisch verstarkte Ausdruck der lebendige Gott nur die Persönlichkeit dieses Wesens zu bezeichnen dient: als allvermögendes Wesen (ens summum) als allweises (summa intelligentia) und allgütiges Wesen (summum bonum)" (usamos, con alguna modificación, la traducción de F. Duque: Transición de los principios metafísicos de la ciencia natural a la física (Opus postumum), Anthropos, Barcelona, 1991, p. 606). 
Para abordar estas preguntas, es especialmente interesante estudiar el tema de la vida de Dios en el curso inédito de Zubiri (prácticamente desconocido por toda la investigación especializada) sobre El problema de Dios (=PD), de 1948-1949. El motivo para utilizarlo es que en él Zubiri desarrolla la idea de Dios como vida con una extensión y un detalle que no se encuentran en ninguna otra parte de su obra. Se trata de un curso de 33 extensas lecciones (¡2089 pp. en total!), recogidas taquigráficamente y luego levemente revisadas por Zubiri. Así, pues, precisaremos la cuestión de la vida divina según Zubiri siguiendo este curso, aunque sin dejar de recurrir a otros inéditos y textos ya publicados del mismo.

Nuestra investigación, entonces, se dividirá en tres apartados. En el primero presentaremos brevemente qué es la vida para Zubiri. En el segundo estudiaremos qué es la vida de Dios para Zubiri, de acuerdo con sus declaraciones en el curso. Finalmente, reconstruiremos esta suerte de teoría del significado de los enunciados teológico-filosóficos a la luz de las ideas maduras de Zubiri, y considerando otras ideas filosóficas tradicionales y contemporáneas.

\section{QUÉ ES LA VIDA}

Para estudiar este tema en Zubiri nos apoyaremos principalmente en el texto "El ser vivo". Este texto formó parte del curso PD. La Primera Parte de ese curso, llamada "El problema de Dios en tanto que problema", se divide en cuatro secciones. La primera de ellas se titula "El ser del hombre", donde trata del hombre como ser vivo. La Introducción y parte de "El ser del hombre" empezaron a ser redactadas posteriormente como libro. La Introducción, levemente mejorada, será publicada en la quinta edición (1963) de Naturaleza, Historia, Dios con el título de "Introducción al problema de Dios"3. El comienzo de "El ser del hombre" llamado "El ser vivo", que mejora y amplía notablemente lo expuesto en el mencionado curso, se publicó, con algunos agregados, en Espacio, Tiempo, Materid . Utilizaremos, entonces, este texto como base, aunque mencionaremos otros textos para precisar mejor la exposición.

Naturaleza, Historia, Dios, Alianza, Madrid, 1987, pp. 393-416.

Espacio, Tiempo, Materia, Alianza, Madrid, ${ }^{2} 2008$, pp. 495-599. 
Zubiri, al comienzo de la Primera Parte de su texto mecanografiado sobre $\mathrm{PD}^{5}$, señala que la vida puede entenderse en dos sentidos. Así nos dice:

En un primer sentido, 'vida' puede significar un modo de ser propio y característico de los vivientes. En su virtud, contraponemos el ser vivo al ser no vivo, al mineral, que tiene otro modo de ser. Aquí aparece, pues, la vida como un modo de ser interno y positivo, común a todo el grupo de seres a que pertenece el hombre. En un segundo sentido, la palabra 'vida' significa el transcurso de los actos vitales. Es lo que queremos decir cuando aludimos a la vida de un personaje cualquiera. Aquí la palabra vida no significa el modo de ser, sino el perfil, el tipo de transcurso efectivo de los actos vitales ${ }^{6}$.

El segundo sentido apunta al dinamismo que poseen los seres vivos. En este sentido, todos los seres vivos poseen un transcurso distinto. El primer sentido, en cambio, apunta al modo o manera de ser de los seres vivos, que Zubiri caracteriza como "autoposesión": la manera como el viviente se enfrenta al medio y a sí mismo. Desde luego, los seres vivos poseen distintas maneras de poseerse (o modos de ser), que Zubiri reduce a tres: vegetal, animal y humana. $\mathrm{Al}$ respecto, Zubiri nos dice:

La vida vegetal consiste en la asimilación. La vida animal [...] es sentir, es sensación, si ustedes quieren. Llegábamos a la vida del hombre cuyo carácter específico y diferencia está justamente en enfrentarse con el mundo circundante, no como un conjunto de estímulos, sino como un conjunto de realidades. Es decir, el hombre responde con el 'es' al choque con el universo físico. La función por la que el hombre ejecuta esto es lo que justificadamente se ha llamado 'inteligencia'.

Este esquema es ampliado y mejorado tanto en la exposición de "El ser vivo" como en el siguiente curso que dicta Zubiri llamado "Cuerpo y Alma” (1950-1951), todo lo cual continúa hasta llegar incluso a Inteligencia sentiente ${ }^{8}$. Este esquema posee dos aspectos a los que hay que atender. Por una parte, hay que atender a la vida desde las acciones y estructuras según las cuales el ser vivo está articulado con su medio. Zubiri realiza aquí un análisis en tres estratos. Por otra parte, Zubiri entiende la

\footnotetext{
5 Que es una mayor elaboración de la 2a lección del curso, pp. 53-54.

6 PD, p. 22.

$7 \quad$ PD, L. 3a , p. 102.

8 Cfr. Inteligencia y realidad, Alianza, Madrid, 1980, pp. 92-96.
} 
vida como un "modo de ser" o, según dirá en escritos ulteriores, como un "modo de realidad". Veremos esto brevemente a continuación".

\subsection{Los tres estratos de la articulación del viviente con su medio}

Zubiri analiza estos tres estratos partiendo desde las acciones vitales. Su análisis va, por así decirlo, desde lo más superficial a lo más profundo. Estos estratos no son independientes, sino que están esencialmente articulados en forma "escalonada", donde el segundo estrato fundamenta al primero y el tercero a los otros dos.

a) El primer estrato es la "estructura suscitación-respuesta". Este estrato intenta describir las acciones de un ser vivo considerado como un "todo" y no desde sus elementos componentes. Así, Zubiri describe acciones como saltar y correr y no, por ejemplo, la contracción muscular presente en estas acciones. Este estrato es el que más se parece a lo que Zubiri en PD llamaba la vida como transcurso. Ahora bien, Zubiri nota que las acciones vitales no surgen espontáneamente, sino que hay algo que las suscita, ya sea del medio interno, ya del externo. Esto que suscita genera un problema, al cual el viviente responde. En escritos ulteriores, como Inteligencia sentiente, Zubiri incorporó a este estrato, entre la suscitación y la respuesta, la modificación tónica ${ }^{10}$. Este primer estrato está a la base de toda esa obra.

b) El segundo estrato es la "habitud-respecto". Zubiri piensa aquí a los seres vivos desde el "modo" o manera como aprehenden las cosas, incluyendo al propio viviente entre ellas. A este modo es a lo que Zubiri llama "formalidad". El ser vivo aprehende contenidos sensibles, pero también formalidades. Por ello, la acción vital no solo queda explicada por las diversas suscitaciones que el viviente pueda sentir, sino también por las diversas maneras de enfrentarse o "habérselas" con las cosas. Zubiri llama a este enfrentamiento "habitud". Hay tres radicales tipos de habitud por las cuales las cosas quedan en distintos "respectos": el vegetar, cuyo respecto formal es el alimento, el puro sentir, cuyo respecto formal es la estimulidad, y el inteligir sentientemente, cuyo respecto

9 Para un análisis más detallado de lo que es ser vivo en Zubiri en las etapas posteriores al curso El problema de Dios, cfr. E. Vargas, "Estudio de la vida en Zubiri: un breve recorrido desde sus primeros cursos extrauniversitarios hasta Inteligencia sentiente", Pensamiento, Vol. 65, No 246, 2009, pp. 713-735.

10 Cfr. Inteligencia y realidad, p. 92. 
formal es la realidad. Esta consideración radical de las habitudes determina la diferencia esencial de las acciones de los vegetales, los animales y del hombre. La habitud, nos dice Zubiri, "no es propiamente un hacer, no es acción, sino una manera primaria de habérselas con las cosas, es actitud" ${ }^{11}$. El vegetal, por ejemplo, no puede aprehender la luz como realidad y, por lo tanto, todo lo que pueda suscitarle la luz se encuentra limitado por su habitud. Las suscitaciones, por ello, pueden ser variables y dependen del medio, pero la habitud no: se tiene o no se tiene. En "El ser vivo", Zubiri dice:

[...] la habitud es el fundamento de la posibilidad de toda suscitación, por parte de las cosas, y de toda respuesta, por parte del viviente. Por esto, mientras la respuesta a una situación es siempre un problema vital, en el orden de la habitud-respecto no hay, ni puede haber, problematismo alguno: o se es vegetal o se es animal o se es hombre. En su estructura más íntima, la vida no es problema: es por lo pronto un factum. Si llamamos conducta al sistema ininterrumpido de las acciones vitales, es evidente que la conducta solo es posible en virtud de la constitutiva habitud del viviente. Es la razón por la que, por muy diversas que sean las biografías de todos los perros, sin embargo todas ellas son caninas. La vida es en esta dimensión un factum primario y radical $^{12}$.

c) El tercer estrato es la "estructura radical del viviente". Se trata ahora de analizar no las acciones del ser vivo, sino los elementos o notas que componen el ser vivo y su "unidad". Para Zubiri, "la vida no es unión sino unidad primaria"13. Esto quiere decir que un ser vivo no consiste en la mera "unión" de sus notas. No es un mero conjunto de sustancias, sino un "sistema" al que llama sustantividad. Propiedades como el perseguir, inteligir, etc., no son resultado de la mera sumatoria de las propiedades de cada una de sus notas componentes. Perseguir, por ejemplo, no se diferencia del huir por las propiedades físico-químicas de los elementos componentes. Los músculos, por ejemplo, se contraen de la misma manera en ambas acciones. Con mayor razón esto ocurre en el inteligir, pues no hay ningún elemento componente -como sus neuronas u otros- del ser humano que intelija. Ahora bien, para Zubiri, las acciones y las habitudes se fundan en las "estructuras". Por una parte, en

11 Zubiri, "El ser vivo", en Espacio, p. 522.

12 Zubiri, "El ser vivo", en Espacio, pp. 520-521.

13 Zubiri, "El ser vivo", en Espacio, p. 572. 
las acciones, la posibilidad de tener ciertas suscitaciones depende esencialmente, por ejemplo, de un determinado tipo de sistema nervioso, que es un componente estructural. Lo mismo deberíamos decir, por otra parte, de las habitudes. El modo como algo se presenta a un ser vivo depende radicalmente de sus estructuras. Una ameba, por ejemplo, está impedida estructuralmente para aprehender realidad.

Pero, la vida puede entenderse también como un modo de ser o realidad. Es lo que veremos brevemente a continuación.

\subsection{La vida como modo de ser o realidad}

Los seres vivos pueden considerarse no solo como un sistema de notas en sus acciones (lo que veíamos antes en tres estratos), sino también por el "modo" en que sus notas son "suyas", es decir, por cómo le pertenecen al sistema vivo. Este aspecto está presente en los tres estratos anteriores. Tanto la suscitación como la habitud y la estructura pueden atenderse desde este aspecto. Así, veíamos que el vegetal, el animal y el hombre son modos de ser vivo. Justamente, en el curso PD, Zubiri llama a esto "modo de ser", no "modo de realidad", por cuanto estos dos conceptos todavía no se distinguían con claridad como en textos posteriores. Pero la idea básica es la misma: la vida como "autoposesión”. Zubiri dice en ese curso que la vida "es la aquiescente y diáfana posesión de sí mismo en la realidad" 14 . Por ello, la vida no es un moverse a sí mismo. El ser vivo ciertamente se mueve, pero es porque ya es un "sî" mismo. Tampoco la vida es un hacerse o, como diríamos actualmente, una autopoiesis. El ser vivo, en cierto modo, se va haciendo, pero es porque ya es sí mismo. Esto Zubiri lo dice espléndidamente en la lección 24:

El vivir no es hacerse a sí mismo a fuerza de alteraciones, sino al revés: estar poseyéndose a sí mismo en todas las alteraciones accidentales o no accidentales que el ser viviente pueda padecer. La mismidad es el supuesto primario de la vida sin el cual el movimiento jamás sería vital. La característica de la vida consiste en no alterar radicalmente al sujeto. El día en que se altera es cuando el sujeto se muere. Mientras hay vida, vivir no consiste en alterarse. Podrá consistir en perfeccionarse o defeccionarse, pero en estos dos movimientos de distinto sentido no acontece nada que sea una alteración radical del sujeto. Con su vida, el viviente se va haciendo mismo, va afirmando

14 PD, L. 2a, p. 98. 
en él su mí, pero no lo va produciendo. Vivir no es moverse, ni tampoco hacer. Justamente al revés. El hacer es vital en la medida en que en él el viviente se está poseyendo a sí mismo. Lo contrario sería, en el sentido más estricto del vocablo, desvivirse. Vivir es, constitutivamente, poseerse ${ }^{15}$.

Ahora bien, esta idea de autoposesión la encontramos de forma más madura en sus últimas obras como El hombre y Dios. Así nos dice:

Todo ser vivo está constituido por una cierta independencia del medio, y un control específico sobre él. Estos dos momentos expresan algo más radical. Es que la independencia y el control expresan que, en sus acciones tanto activas como pasivas, el viviente actúa no solo por las notas que posee sino también en orden al sistema que constituyen. Ciertamente, toda sustantividad, aunque sea meramente mineral, puede envolver en sus actuaciones la totalidad del sistema, pero el mineral no tiene ninguna acción ordenada a la totalidad del sistema en cuanto tal. Este momento es el momento radical y formalmente exclusivo de la vida. Se vive por y para ser sí mismo. Es decir, el viviente es un 'sí mismo', un autós's .

Este modo de realidad como autoposesión posee diversos grados, desde un virus o una célula hasta el hombre, que se autoposee como realidad. En los seres vivos naturales, su modo de realidad depende de sus estructuras. Este es el esquema básico desde el que Zubiri trata de entender la vida. El problema se nos presenta ahora cuando tratamos de entender la vida de Dios. Zubiri afirma que Dios es vida. ¿Cómo entender esto desde lo ya dicho? Es lo que hay que presentar a continuación.

\section{LA VIDA DE DiOS}

En el curso PD, la cuestión de la vida de Dios es tratada con morosidad en la $24^{\text {a }}$ lección $^{17}$. Siguiendo una división que hace Zubiri en las "Fichas" que lleva al curso, dividiremos esta parte en dos secciones. En primer lugar, veremos en qué consiste la acción vital de Dios. En segundo lugar, veremos a Dios como realidad plenaria, lo cual permite aclarar mejor de qué tipo particular de acción vital es la vida de Dios. En su "Ficha" que lleva al curso le llama a esta sección "Realidad plena-

PD, L. 24, p. 1401-1402.

16 Zubiri, El hombre y Dios, Alianza, Madrid, 22012, pp. 30-31.

17 Sobre todo entre las pp. 1391-1446. 
ria”. Dado que este curso permanece inédito y para hacer contrastable nuestra posterior reconstrucción, expondremos ahora las afirmaciones fundamentales de Zubiri sobre el tema.

\subsection{La acción vital de Dios como autoposesión}

Ante todo, veamos qué es Dios para Zubiri. Para Zubiri, ya en el curso PD, realidad es el "de suyo". La inteligencia es la facultad de aprehender la realidad. En esta realidad inteligida, Zubiri distingue entre "las cosas reales" (lo real) y la "unidad" de esas cosas reales, su momento de realidad. Esta distinción le permite entender a Zubiri los diversos tipos de fundamento u origen de las cosas. La ciencia, por una parte, estudia el fundamento de las cosas reales. La filosofía, por otra parte, estudia el fundamento de las cosas reales en tanto reales. Ese fundamento es Dios. Así Zubiri nos lo demuestra:

Si hay alguna realidad, inexorablemente tiene que haber una realidad que sea la primera por razón de la realidad de las cosas. [...] Es decir, si la primera realidad tiene que ser razón y causa de la realidad en tanto que realidad, inexorablemente tiene que ser la realidad plenaria, la plenitud de lo real sin negatividad, porque, si no, nos plantearíamos otra vez la pregunta del por qué18.

Todas las cosas reales, en definitiva, son creadas por Dios, pero no solo porque Dios les haya dado origen a todas las cosas reales en el pasado, sino porque, desde siempre, las sostiene "en tanto que realidades". Zubiri intenta una prueba que demuestre la existencia de Dios no por la necesidad de una realidad plena en el origen del universo, o en algunas ocasiones especiales del hombre, sino en cada instante. Por ello dice que "la razón formal de la existencia de Dios no es que el mundo tenga o no comienzo, sino que esté siendo"19.

Según Zubiri, Dios es realidad fundamental, natura naturans: "el ente fundamentante es la realidad primera del universo, es natura naturans, una naturaleza naturante como raíz del universo" ${ }^{20}$. Se lo llama justificadamente Dios, "porque es una realidad fundamental de la realidad misma y de la mismidad vital en cuanto tal" ${ }^{21}$. Dios, en este sentido, "es

\footnotetext{
PD, L. 22, p. 1313.

PD, L. 23, p. 1352.

PD, L. 24, p. 1391.
}

21 Ibid. 
una mismidad absoluta, un sí mismo absoluto, bien entendido que aquí ser sí mismo no significa -me refiero a cualquier acto vital, no solo al divino, sino a cualquier acción- una inerte entidad como la que pueda tener un soporte sobre el cual pasan muchas vicisitudes, o un sujeto que pasivamente va quedando idéntico a través de las mutaciones del universo" 22 . Dios es, entonces, una mismidad absoluta, pero no inerte sino accionante o dinámica, como dirá Zubiri en sus últimos escritos. Es una acción que Zubiri califica de vital.

Ahora bien, hay que meditar en qué consiste la acción vital de Dios. Veíamos antes que en los seres vivos podíamos distinguir, por un lado, sus acciones y estructuras y, por otro lado, su modo de ser o realidad como autoposesión. En el caso de Dios, esta distinción no parece posible. Dios no es una entidad que luego cambie, sino que posee una profunda unidad. En este sentido, hay en Dios una implicación entre lo entitativo y lo operativo en una "positiva acción”. Dice Zubiri:

la mismidad no consiste en estar siendo lo mismo a pesar de las variaciones, sino que consiste en la acción positiva de poseerse en su propia realidad. Esto es, la mismidad como acción. En este sentido radical de la mismidad como acción es aquel en que esencialmente consiste la vida. Vivir no es, primariamente, moverse. Justamente al revés, el movimiento es vital cuando lo que acontece no altera al ser viviente, sino lo mismifica, si se me permite el neologismo ${ }^{23}$.

Vivir, según Zubiri, como hemos visto, no es tanto moverse o hacerse a sí mismo, sino más bien autoposeerse a través de los propios actos.

El ente finito "que tiene acción de vivir es, constitutivamente, un ser viviente, un ente cuya naturaleza completa ejecuta la acción que consiste en poseerse a sí mismo" ${ }^{24}$. Por ello puede decirse que la vida es mismidad, y que

la mismidad es una acción, es un acto que emerge radical y constitutivamente de la naturaleza del viviente. Es en este sentido un acto primario. El entrar en sí mismo, la mismidad, no es un [mero] entrar, sino un estar en sí mismo. La mismidad, el mí, no es resultado, sino principio mismo constitutivo de la vida ${ }^{25}$.

\footnotetext{
Ibid.

PD, L. 24, p. 1401.

PD, L. 24, p. 1403.

25 Ibid.
} 
Por tanto, cabría decir que Dios mismo es activo en el preciso sentido en que es eminentemente sí mismo:

es acción, es mismidad, es la acción de su mismidad [...] Dios es acción vital. Mientras operamos con el concepto de vida [...] montado sobre la idea del movimiento y del hacer, siempre parecerá paradójico que Dios sea vida. Dios es vida en el sentido más radical del vocablo, porque es la mismidad absoluta ${ }^{26}$.

Ahora bien, ¿en qué consiste con mayor rigor la acción vital divina? La respuesta a esta pregunta es lo que Zubiri llamara Dios como realidad plenaria.

\subsection{Dios como realidad plenaria}

Decir que la acción vital de Dios consiste en autoposesión, para Zubiri, es algo impreciso porque, en definitiva, también vegetales y animales son vivientes en tanto poseen este modo de ser. Por tanto, habría que especificar qué es lo propio de la autoposesión divina, de su acción vital. Así Zubiri lo señala:

decir que Dios como mismidad absoluta es acción vital y, por tanto, es vida, no es más que hablar de la mitad de la cuestión. Es menester que digamos en qué consiste la peculiaridad de la acción vital divina [...] Es Dios acción vital en el sentido de que es plena posesión de sí mismo. Es preciso definir con un poco más de rigor qué es esta plenitud de posesión ${ }^{27}$.

¿Qué es lo propio de la autoposesión divina? Zubiri aborda este problema desde el punto de vista negativo y positivo. Veámoslo por partes.

a) Desde el punto de vista negativo, hay que decir que la realidad divina "no" consta de propiedades. Y si Dios no tiene propiedades,

es absolutamente quimérico e imposible que la palabra propiedad atribuida a Dios tenga ningún sentido que no sea el de constituir [...] la realidad en cuanto tal. En manera alguna se trata de volcar sobre Dios un montón de propiedades que no sabemos de dónde proceden $^{28}$.

26 Ibid.

27 PD, L. 24, p. 1404.

28 PD, L. 24, p. 1405. 
Para Zubiri, el que Dios no tenga propiedades quiere decir que es una realidad simple, que es sí mismo simplemente, plenariamente, vale decir, sin negatividad que lo limite:

la propiedad en Dios tiene que venir inexorablemente implicada en el puro y simple hecho de que Dios es realidad plenaria. Dicho en otros términos: las presuntas propiedades físicas de Dios son en cierto modo puras concreciones que el intelecto humano se verá forzado a admitir en Dios; pura y simplemente, en cuanto entitativamente Dios es realidad plenaria, la realidad, que no va perforada por ninguna dimensión de negatividad y ante la cual el intelecto humano no solo no se plantea, sino que por razón del objeto no puede constitutivamente plantearse la pregunta de un ulterior por qué [...] Por consiguiente, las propiedades que podemos descubrir en Dios son propiedades que le pertenecen en tanto y en cuanto es justamente la realidad plenaria. Aplicada esta consideración al acto vital, nos encontramos con no escasas dificultades de superar, si queremos entender a Dios como acción vital en el sentido de realidad plenaria ${ }^{29}$.

Para explicar esto, Zubiri analiza el acto vital de un ser vivo cualquiera: "evidentemente, en un acto vital cualquiera -el mío, el de un gato, de un árbol [...]- nos encontramos siempre con un ser viviente que ejecuta la acción de vivir y que, a consecuencia de la acción de vivir, se encuentra en un estado determinado a consecuencia de ese acto" ${ }^{30}$. Hay, pues, que distinguir entre sujeto, acción y estado: "por razón del sujeto, [...] el viviente puede morir; como acción, la acción de vivir puede conducir a distintos estados, etc." ${ }^{31}$. En el caso del hombre esta distinción es más clara: "el hombre vive en buena medida desde sí mismo y, además, vive para sí mismo. Desde sí mismo: ahí está el sujeto de su acción; para sí mismo: ahí está la versión de la acción"32. Pero en el caso de Dios la

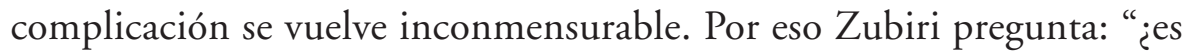
posible aplicar este esquema a la idea de una acción vital que sea realidad plenaria?" Y responde: es "entitativamente imposible"33. Es imposible admitir en Dios

\footnotetext{
PD, L. 24, p. 1406.

PD, L. 24, p. 1407.

Ibid.

Ibid.

Ibid.
} 
este esquema de la vida, no solamente por lo que este esquema tiene de vida, sino por lo que envuelve justamente de distinción. Distinción, cualquiera que sea el orden y su grado [..., la hay entre el sujeto, la acción y el estado. Sin embargo, esta distinción es absolutamente intolerable en algo que pretende ser realidad plenaria, porque donde hay distinción el ente que la posee está intrínsecamente perforado por una negatividad. Cada elemento no es el otro. Si no, no habría distinción; sería una perfecta identidad. Es absolutamente imposible, pues, que el ente divino como realidad vital, como acción vital plenaria, pueda entrar en este esquema. No puede haber en la acción vital divina distinción en este sentido entre un sujeto, una acción y un estado. Ahora bien, esto queda excluido por el concepto de la realidad plenaria $[\ldots]^{34}$.

La primera consecuencia de esto es que, aunque parezca paradójico, "Dios no es un ente viviente, no es un ser que vive, sino simplemente la vida misma [...] Dios no es un viviente" ${ }^{35}$. Si fuera un viviente, entonces habría "alguna distinción entre su sujeto, su acción y su estado, o sería el resultado de la conjunción de estas tres dimensiones, o bien sería su principio anterior a la acción"36. De ser así,

la realidad de la acción vital del ente divino sería una realidad, en cierto modo, sida: sería lo que ese sujeto tiene o puede tener de ser. Desde el momento en que es sida, no es la realidad plenaria, porque va perforada por la negatividad. No puede concebirse la acción vital divina como una acción que emerge, ni siquiera exhaustivamente, de sus propias posibilidades. La acción vital divina es inemergente, es puramente subsistente ${ }^{37}$.

Por esto es por lo que Dios no es un viviente, sino un ser por completo simple: "lo primero que, negativamente, nos aparece en la acción vital divina es que es, constitutivamente, simple. Simple no significa [...] meramente pobre [...] Se trata justamente de todo lo contrario"38. Si hay una simplicidad por pobreza, podría haber también, inversamente, una simplicidad por riqueza. Sería

\footnotetext{
PD, L. 24, p. 1408.

PD, L. 24, p. 1409.

Ibid.

PD, L. 24, p. 1410.

Ibid.
} 
el carácter formal de la riqueza de un acto. En esta simplicidad de la vida divina $[\ldots]$, nos aparece la inexistencia de la identidad y la no identidad entre el sujeto, la acción y el estado [...] Ahora bien, como no se trata de la pobreza, sino de la plenitud de la realidad, quiere decirse que esa dimensión puramente negativa lo único que con su negación hace es circunscribir el área dentro de la cual, positivamente, es menester pensar ${ }^{39}$.

En definitiva, desde el punto de vista negativo, Dios es un ser simple en tanto que no tiene propiedades. No puede distinguirse en Él entre su sujeto y sus acciones.

b) Sin embargo, decir que en Dios no se distingue entre el sujeto y la acción, no es decir nada obvio ni ostensible. ¿Qué significa la acción vital divina en términos positivos?:

En esta dimensión positiva del problema es donde, fácilmente, puede deslizarse la mente humana en dos direcciones [...] La mente humana no puede pensar, generalmente, una identidad, como no sea la formal 'A igual A', más que por vía de identificación. Naturalmente, decir que $\mathrm{A}$ y $\mathrm{B}$ son dos realidades idénticas consiste en decir que, aunque parece que $\mathrm{A}$ y $\mathrm{B}$ son distintos, no son en realidad más que uno; pero es muy diferente decir que $A$ y $B$ son idénticos porque $A$ no se parece a $B$, pero es $B$, que decir, al revés, que $B$ no se parece a $A$, pero en realidad es $A$. En el primer caso, el resultado de la identificación será que A y B son A, y en el segundo que A y B son B. Como tenemos tres términos, sujeto, acción y estado, es menester pensar muy detenidamente adónde y en qué línea tenemos que apuntar para la identificación que nos ponga ante los ojos la radical originalidad del acto vital divino ${ }^{40}$.

Una alternativa "cómoda" es la de "decir que la realidad a que se refieren [los tres términos] es simple y decir que sobran algunos de esos elementos y que no hay más que uno [...] La simplicidad se obtendría por simplificación” ${ }^{41}$. Dios sería simple por simplificación de sus propiedades a una sola. Sin embargo, que la vida divina sea simple, conviene aclararlo, no implica que tenga

nada de simplificada. La simplificación es el recurso de la pobreza de un concepto. La simplicidad es cosa más difícil de conquistar en el

39 PD, L. 24, p. 1411.

40 PD, L. 24, p. 1412.

41 PD, L. 24, p. 1413. 
orden de los conceptos. Por esta vía de simplificación, que es la vía de la pura identificación, digo que hay dos pendientes por las cuales se desliza fácilmente el intelecto ${ }^{42}$.

Ahora bien, para Zubiri han existido dos grandes posibilidades de simplificación de la realidad divina. La primera apunta a la vía hegeliana:

Por un lado, podremos decir, puesto que no se distinguen el sujeto, la acción y el estado, que cuanto hay en la acción vital divina y en el estado [...] consecutivo, en realidad no es nada distinto del sujeto. En este sentido, Dios como realidad absoluta, como acción vital absoluta, sería sujeto absoluto. Fue la posición [...] de Hegel. Ahora bien, esto es entitativamente imposible [...] Si ese sujeto se piensa sin ninguna acción, no sería, en definitiva, nada; sería algo perfectamente indeterminado ${ }^{43}$.

Empero, la acción vital divina no es radicalmente indeterminada ni determinada. Por ello mismo, Dios no es sujeto absoluto.

Por otro lado, hay una segunda vía: suprimir el sujeto y quedarse con la pura acción, el estar divino, una acción subsistente que no necesitaría de sujeto alguno. Esto es también entitativamente imposible. Si la primera vía es la panteísta, la segunda es la de un puro actualizar, una acción que es un hecho: Dios como el que hace. Esta última puede intentar mejorarse cuando se dice que el estar siendo Dios como está siendo arranca de una "inexorable necesidad interna" ${ }^{44}$.

En todo caso, piensa Zubiri, la vía de la simplificación no es recomendable. No obstante lo cual,

nos conduce, negativamente, a hacer ver que la mismidad y la acción absoluta de Dios están allende el estar y allende el hacer. No consiste en otra cosa sino en ser, en estar siendo. Por tanto, es menester eliminar de la idea de la acción vital divina cuanto hay de referencia a un sujeto y a un estado y esta idea de la acción subsistente no se obtiene substrayendo elementos a la acción vital, sino, al revés, superando la disyunción entre sujeto y estado por la perfección interna de la acción vital. Esta es la única vía por la cual puede concebirse una acción vital como realidad plenaria ${ }^{45}$.

\footnotetext{
Ibid.

Ibid.

PD, L. 24, p. 1414.

45 PD, L. 24, pp. 1415-1416.
} 
Dios, pues, no es un viviente, sino la vida misma; "es vida subsistente, en su perfecta simplicidad, con una inexorable necesidad" 46 . Dios tampoco tiene una vida causal, como sí la tienen los vivientes finitos, ni siquiera en el sentido de que fuera causa de sí mismo. Si Dios es vida subsistente, es que en él se da la sustantividad del vivir ${ }^{47}$. Por lo mismo, dice Zubiri, Dios existe plenariamente, "por una elevación progresiva de cuanto hay de existencia sustantiva en los entes finitos, para centrarla en la pura y simple acción que consiste meramente en existir con plena sustantividad" 48 .

Como acción vital, en fin, la acción divina es íntima, pero no por ello aislada, sino intrínsecamente creadora ${ }^{49}$. Es la acción de un individuo absoluto, contra lo que digan los panteísmos ${ }^{50}$. Es la acción de la vida perfecta $^{51}$. Es la acción de la vida infinita ${ }^{52}$. Es la acción de la vida inconmensurable, inmensa ${ }^{53}$.

\section{UNA RECONSTRUCCIÓN}

Ahora bien: ¡cómo compatibilizar las primeras tesis de Zubiri -aquellas sobre los seres vivos- y las que acabamos de exponer -desarrolladas para predicar la vida de Dios-? Puesto que Dios no tiene las notas de animales y plantas, ¿resulta plausible el argumento zubiriano según el cual Dios es vida? ¿Cómo salvar las diferencias entre la vida intramundana y una vida extramundana? Porque la vida intramundana es justo aquella en la que cabría distinguir, según el lenguaje de Zubiri, entre la vida como transcurso de acciones fundado en determinadas estructuras y la vida como modo de ser o realidad. Para tratar mejor estas cuestiones, por lo demás, será útil aquí recurrir a tesis tradicionales y contemporáneas acerca de la significatividad que caracteriza al lenguaje religioso, teológico e incluso filosófico en relación al tema de la vida intramundana y de la vida de Dios.

\footnotetext{
PD, L. 24, p. 1416.

PD, L. 24, p. 1417.

PD, L. 24, p. 1419.

PD, L. 24, p. 1420.

PD, L. 24, p. 1421.

PD, L. 24, pp. 1421-1433.

PD, L. 24, pp. 1438-1439.

33 Dicho sea de paso, en el curso de 1968 sobre El hombre y el problema de Dios, la vida de Dios aparece como fundamento del poder de lo real, como realidad esencialmente existente, como persona inteligente y volente, y como realidad fundante del mundo y de la vida personal (=esencia abierta) del hombre.
} 


\subsection{De vita Dei}

Zubiri, creemos, procede de manera análoga a como lo hace Tomás de Aquino cuando se enfrenta con la cuestión de la vida de Dios ${ }^{54}$. La estrategia de Tomás consiste en partir de lo que nos es más conocido: "por los seres que, manifiestamente, viven, podemos saber quiénes viven y quiénes no viven" ${ }^{55}$. Es ostensible que los animales viven. Vivir, para ellos, es moverse, tener movimiento, pero no cualquier movimiento, sino un movimiento de suyo (ex se), no por otro ( $a b$ alio). Así, el que no se mueve no está vivo, está muerto. El viviente es el que se mueve por sí mismo, sea propiamente (esto es, por un acto imperfecto), como cuando se mueven en el sentido del desplazamiento o del crecimiento o del envejecimiento, sea impropiamente (o sea, por un acto perfecto), como cuando se mueven en el sentido de inteligir o sentir. El que no se mueve por sí mismo, en cambio, no vive, sino solo por cierta semejanza (per aliquam similitudinem). Zubiri hace algo semejante, aunque no idéntico. $\mathrm{Si}$, como dice Tomás, "por los seres que, manifiestamente, viven, podemos saber quiénes viven y quiénes no viven”, entonces hay que determinar tan precisamente como se pueda cuál es el sentido más manifiesto de la vitalidad. Una alternativa hermenéutica es la de entender que la vida más manifiesta es la vida en el primer estrato, la vida como transcurso de acciones fundadas en ciertas estructuras. Allí se advierte ya un criterio para distinguir entre los seres vivos y los no vivos. La vida sería aquello que es común a vegetales, animales y seres humanos. Empero, esta es una afirmación muy parcial y extremadamente inadecuada. La vida que tenemos más a mano es la nuestra. De ahí parece emerger la idea de vida como modo o forma de realidad. Porque también se advierte que la diversidad de acciones y estructuras vitales funda modos esencialmente diversos de ser vivo. Y que entre dichos modos vitales hay gradación o, mejor dicho, jerarquía, desde la vida propia o imperfecta -en los términos tomistas- hasta la vida impropia o perfectamente tal.

En segundo lugar, Tomás cree que vivir es un predicado sustancial, no meramente accidental. Lo que significa que los seres vivientes viven no solo por las operaciones que ejecutan, algo que es derivado y aparente, sino también por la naturaleza de la sustancia que son y por la cual actúan como actúan. De guisa que el automovimiento queda precisado

54 Cf. STh I, q. 18, a. 1-4.

55 Cf. STh I, q. 18, a. 1, c. 
como una operación de ese ser que es el vivir. Dicho de otro modo, la vida de una cosa se exterioriza en "moverse por sí misma (movere seipsum)" "Z6 . Zubiri procede similarmente. No son solo acciones y estructuras las que definen la vitalidad, sino sobre todo el modo de ser real que ellas fundamentan. Sin embargo, la vida, por una parte, como hemos visto, no es automovimiento, sino autoposesión. Por otra parte, la vida no es para él un predicado sustancial, sino sustantivo: es un sistema de notas (o incluso de una sola nota) en unidad coherencial primaria. Y uno que no se caracteriza por el automovimiento como por la mismidad, por una autoposesión plenamente coherente, por ese carácter excepcional en que el viviente es irrebasablemente autós. Vivir, desde esta perspectiva, es no solo un acto, sino el modo más sustantivo de ser: es autoposeerse perfecta, absolutamente.

En tercer término, Tomás piensa que la vida está en Dios “máxima y propiamente (maxime proprie)" ${ }^{57}$. El moverse por sí mismo admite grados, y por ello la vida puede ser más o menos perfecta. Desde un instrumento (que ejecuta la acción que le imprime el agente), pasando por plantas (que se mueven, desarrollan y declinan, pero no según forma y fin, sino estrictamente determinadas por su propia naturaleza) y animales (que se mueven de acuerdo con su forma por sus sentidos), la vida va perfeccionándose. Pero ninguno de estos seres establece el fin de su operación o movimiento, sino que él está impreso en su naturaleza. Fijar el fin de la operación es lo que hace la razón y el intelecto: es el mayor automovimiento, "el modo más perfecto de vivir (perfectior modus vivendi)" 58 . He aquí la superioridad de la vida humana respecto de la animal y la vegetal: en el hombre la fuerza intelectiva (virtus intellecti$v a)$ mueve a las potencias sensitivas, y estas a los órganos ejecutores del movimiento. Pero, en el hombre la perfección no es total. Todavía hay en él cosas grabadas indeleble e inmutablemente en su naturaleza, como los primeros principios (prima principia) y el último fin (ultimus finis). En este sentido se mueve por sí mismo, pero no plenamente, sino que en parte sigue siendo movido por otro. De manera que aquel ser cuya naturaleza y orientación sean puro inteligir (intelligere), sin estar determinado por otro (non determinatur ab alio), posee la mejor vida. Es,

56 Cf. STh I, q. 18, a. 2, c.

57 Ibid.

58 Cf. STh I, q. 18, a. 3, c. 
por supuesto, lo que ocurre con Dios, cuyo intelecto es "perfectísimo y siempre en acto (perfectissimus, et semper in actu)" ". Vivir no es en Dios sino inteligir. Por ello, todo lo que él ha hecho está en él inteligido por él. Así, puede llegar a decirse que "todas las cosas son en él la misma vida divina (omnia sunt ipsa vita divina)" o, aun más ceñidamente, que en Dios "es lo mismo su intelecto, lo inteligido y su inteligir (est idem intellectus, et quod intelligitur, et ipsum intelligere eius)" ${ }^{60}$. Para Zubiri, por su parte, también la vida divina es la máximamente propia. Si el autós está graduado o, mejor, jerarquizado, entonces la mismidad en Dios habrá de ser la máxima o mejor. Y lo será porque -en esto Zubiri coincide con Tomás- la perfección de todo viviente pende de que su finalidad no esté impresa en su naturaleza, sino más o menos establecida por él mismo. Es justo lo que acontecería con Dios. Su perfección es total. Nada hay en él grabado indeleble e inmutablemente. Su principio y su finalidad no le están fijados heterónomamente, sino que brotan de su plena y coherente autoposesión. En este sentido, se posee a sí mismo por sí mismo, y no por otro. Además, Zubiri piensa que la vida de Dios es tanto intelectiva como también volitiva e incluso (como aparecerá tardíamente en su obra) sentimental. Por ello, Zubiri reformularía el dicho tomista, afirmando no solo que en Dios es lo mismo "su intelecto, lo inteligido y su inteligir", sino que en él es lo mismo su sentimiento, lo sentido y su sentir, así como es lo mismo en él la volición, lo querido y el querer. Vivir, así, sería en Dios una autoposesión inteligente, sentimental y volitiva, enérgica y perfectamente actual.

Zubiri procede como Tomás cuando predica la vida de Dios. Pero lo hace, claro, mutatis mutandis. Que Dios sea vida no quiere decir para Zubiri que sea el principio del movimiento máxima y propiamente. Sino que es sí mismo máxima y propiamente, el ser o la realidad autoposeída par excellence. Dios vive, pero no vive por desarrollar operaciones fisiológicas como nutrirse, ni por tener sensaciones, ni por poseer estructura celular o código genético, sistema nervioso o parasimpático, etc. Sin embargo, Dios tiene acciones o dinamismos (por ejemplo, allí están, ad intra, sus procesiones trinitarias, y ad extra, la creación y la encarnación ${ }^{61}$ ). Por eso Dios no tiene, sino que es acción vital. Dios vive, entonces, en el

\footnotetext{
59 Ibid.

60 Cf. STh I, q. 18, a. 4, c.

61 Cf. El problema teologal del hombre: Cristianismo, Alianza, Madrid, 1997, pp. 233-234.
} 
sentido de que la vida, considerada eminentemente, es Dios. Y la vida en Dios no admite distinguir entre sujeto, acción y estado, pues es siempre y solo la plenitud de ser sí mismo. Vale decir, Dios está vivo, pero en un sentido parcialmente idéntico y parcialmente distinto de aquel según el cual está vivo cada cual. ¿Quiere esto decir que Zubiri hace suya la vía tomista de la analogía cuando predica la vida de Dios?

El asunto es espeso. Hay quien piensa-siguiendo en esto a medievales tardíos como Escoto y Ockham, y a empiristas británicos recientes como Ramsey y Crombie- que la teología es informativa y procede -en una proporción decisiva- unívocamente. Lo que es tanto como decir que en ella se usan palabras ordinarias en sus sentidos ordinarios, es decir, literalmente, si bien en combinaciones, circunstancias y grados inusuales. Por lo mismo, si el lenguaje teológico pretende capacidad informativa, entonces su proceder no literal sino analógico -el que otorga nuevos sentidos a palabras ordinarias- tendría que ser más bien excepcional o residual ${ }^{62}$. Empero, según creemos, Zubiri calificaría de no literales a los predicados (y enunciados) en los que el significado está cualificado por combinaciones, circunstancias y grados inusuales, como acontece por antonomasia con la vida de Dios. Es lo que precisamente quiere decir que respecto de Dios se use una palabra ordinaria como vida: que no se usa, al menos no enteramente, en su sentido ordinario o simplemente literal, sino con desplazamientos cuantitativos y/o cualitativos. Esto llevaría a pensar que declarar la vida de Dios es una manera analógica de hablar, sea religiosa, sea teológicamente. De hecho, lo que más se ha comentado en Zubiri no es tanto su afirmación de que Dios es vida, sino su explícito tratamiento de la analogía a propósito de la absolutidad, la por él denominada "analogía de lo absoluto". Esta analogía, que Zubiri conquista recién en su madurez filosófica, no es otra cosa que la simultánea afirmación de que mientras el hombre es relativamente absoluto, Dios en cambio es realidad absolutamente absoluta ${ }^{63}$.

Todo el punto radica en el criterio usado para distinguir a la analogía. Pues si analógico es aquel modo de hablar literal pero cuantitativamente

62 Vid. R. Swinburne, "Las palabras de la teología”, en E. Romerales (ed. y trad.), Creencia y racionalidad. Lecturas de filosofía de la religión, Anthropos, Barcelona, 1992, pp. 125-160.

63 Vid. sobre la analogía de lo absoluto la nueva edición de El hombre y Dios, pp. 186189 y $474-476$. 
diverso o acrecentado, es decir con una mera diferencia de grado o cantidad, entonces no sería analógica la manera metafórica de hablar. Pero si la analogía supone un modo de hablar ya no literal sino cualitativamente diferente, vale decir con una diferencia no solo cuantitativa sino estricta y formalmente cualitativa, habría que decir que la metáfora es también analogía $^{64}$. Y que, por ende, la vida de Dios sería una forma analógica y, eo ipso, metafórica de referirse a la divinidad, y una que -si la metáfora es una manera legítima y a veces irreductible de decir ciertas cosas difíciles de decir- no por ello pierde (ni menos carece de) significatividad. Trataremos, para terminar, de hacer ver que este es el caso en Zubiri.

\subsection{Carácter y elementos del lenguaje acerca de la vida divina}

Para ello, es necesario reconstruir las fragmentarias reflexiones zubirianas, tomando - como no puede ser de otro modo-opciones conjeturales y discutibles. En este sentido, si se consideran las ideas maduras de Zubiri, se vislumbra una teoría que posibilita otorgar significado metafórico a los enunciados religiosos y teológicos y, en particular, a la afirmación de la vida divina ${ }^{65}$.

64 Cf. E. Romerales, Concepciones de lo divino. Introducción a la teología filosófica, Publicaciones de la Universidad Autónoma de Madrid, Madrid, 1996, pp. 74-82. Entre los predicados (y enunciados) teológicos Romerales distingue predicados (y enunciados) literales (tanto positivos como negativos) -que proporcionan comprensión directa-, literales pero a la vez cualificados -que dan comprensión indirecta o analógica-, y no literales o metafóricos -que solo proporcionan comprensión metafórica o bien ninguna comprensión-; incluso hay enunciados paradójicos rescatables en algún sentido. Un término metafórico es precisamente 'vida', el cual no se distingue de un término literal solamente por la cantidad (es lo que ocurre con un término cualificado), sino también por la cualidad. 'Así, 'vivo' aplicado a Dios no difiere de 'vivo' en grado, sino en cualidad. Verosímilmente hay muchos aspectos cualitativos que diferencian la 'vida' divina de la vida animal o vegetal. Por ejemplo, lo que define la vida es la capacidad de reproducción, pero Dios no se puede reproducir. Todo ser vivo ha nacido y puede morir $-\mathrm{y}$ morirá-, pero Dios no, etc. Lo mismo vale para la autorregulación, el crecimiento y la nutrición. Y decir que Dios no es 'vida', sino 'fuente de vida', es solamente dar otro nombre a lo desconocido; es pretender pasar un término metafórico por uno cualificado, lo cual es ilícito pero asaz frecuente entre los teólogos" (p. 75). Los términos metafóricos son usuales en el lenguaje religioso, mientras que los cualificados lo son en el lenguaje teológico, incluso a veces el mismo término puede funcionar en el lenguaje religioso y en el teológico, si bien con diferente significado (p. 76).

65 Dicho sea de paso, esta vía de la metáfora es usual recientemente: vid. P. Tillich, Teología sistemática, vol. 1: La razón y la revelación. El ser y Dios, trad. D. Sánchez- 
Para Zubiri, Dios es el núcleo de la religión o, dicho más estrictamente, la idea definidora de un campo religioso ${ }^{66}$. Las muy diversas ideas de Dios son simples aprehensiones de una realidad-fundamento. Estas ideas no son representativas, como si Dios fuera un objeto meramente intramundano, sino que son aprehensiones campales direccionales que indican una vía interminable:

no se trata de objetos, sino de la dirección-hacia. En esta línea del 'hacia' es donde se inscribe formalmente el pensar religioso ${ }^{67}$.

Aunque tengan un momento representativo, las ideas de Dios son prevalentemente direccionales. Las ideas de Dios son simples aprehensiones y, como tales, construcciones libres y creadoras. Pero por su máxima direccionalidad y mínima representatividad, son unas construcciones peculiarmente oscilantes. En ellas, es inconmensurable la diferencia entre la simple aprehensión y aquello que es simplemente aprehendido. Se llega a la idea de una realidad absolutamente absoluta a partir de la idea de las realidades relativas. El movimiento que parte de realidades relativas permite postular una realidad otra como fundamento del poder de lo real. Es la libre postulación de una realidad absolutamente absoluta y transcendente que sí puede desempeñar campalmente una función fundamental para con todas las cosas relativas del campo. Así, la simple aprehensión de Dios es postulada prescindiendo positivamente de los rasgos de las realidades campales relativas. La idea de Dios es oscilante porque fluctúa entre las realidades relativas de las que se diferencia pero

Bustamante, Sígueme, Salamanca, ${ }^{4} 2001$ : "no puede existir ninguna duda de que toda aserción concreta acerca de Dios ha de ser simbólica, ya que una aserción concreta [por ej. la de Dios como viviente] es la que utiliza un segmento de la experiencia finita para decir algo acerca de Dios. Transciende el contenido de este segmento, aunque también lo incluye. El segmento de la realidad finita que se convierte en vehículo de una aserción concreta acerca de Dios, queda afirmado y al mismo tiempo negado. Se convierte en un símbolo, ya que una expresión simbólica es aquella cuya significación propia es negada por aquello hacia lo que apunta. Pero asimismo es afirmada por él, y esta afirmación es la que confiere a la expresión simbólica una base adecuada para apuntar hacia más allá de sí misma” (p. 308; vid. pp. 307, 309, 311, 315 y 320-323).

66 En lo que sigue, nos basaremos en E. Solari, La raíz de lo sagrado. Contribuciones de Zubiri a la filosofía de la religión, RIL editores, Stgo. de Chile, 2010, pp. 262ss.

67 Zubiri, El problema filosófico de la historia de las religiones, Alianza, Madrid, 1993, p. 136. 
a partir de las cuales ha sido forjada, y esa dirección absoluta y transcendente que jamás alcanza pero a la cual apunta.

Por lo mismo, las ideas de Dios tienen ingredientes ficticios, perceptuales y conceptivos. Las simples aprehensiones de Dios son no solo diversas sino también mixtas. Aplicar de Dios la vida, en este sentido, es aplicarle un concepto y un ficto, pero también un percepto. La idea según la cual Dios es vida es una simple aprehensión abstracta de que sería el poder de lo real: es un concepto. Además, como esta realidad reduplicativamente absoluta es infinita, su idea es la libre reconfiguración de las notas campales que caracterizan intramundanamente a la vitalidad: es un ficto que permite aprehender, mediante imágenes y siguiendo alguna determinada línea intelectiva, cómo sería el poder de lo real. Y por ello, en este ficto está presente la vida percibida, pues en la noción de Dios vivo algo hay del percepto de la vida. Claro, la idea de Dios como vida no se identifica con el percepto campal de vida, pero sí que depende de él. Y es que para referirse direccionalmente a la realidad absolutamente absoluta y transcendente como una realidad viva hay que incluir a la vida percibida, aunque abstracta (dejando de lado positivamente sus rasgos intramundanos) y ficticiamente (reconfigurando con libertad sus notas).

Así, la vida de Dios es sobre todo una metáfora. La metáfora de la vitalidad divina permite aprehender distanciadamente qué sería el fundamento del poder de lo real. Dios como vida es un ficto que, apoyado en el percepto de la vida, tiene una función abstracta, vale decir, conceptual. Más precisamente, cuando se dice que Dios es vida se dice que lo que el fundamento del poder de lo real sería es un concepto al que se llega mediante la postulación ficcional de cómo sería, y que para hacer esto, el percepto campal de vida del que se prescinde sirve, sin embargo, de apoyo para apuntar direccionalmente a una realidad absoluta, infinita, extramundana y transcendente. Dios como vida, entonces, es una idea de Dios predominantemente ficticia o fantástica, lo que no significa que sea una ficción, claro, sino que es una idea fuertemente metafórica que incluye un momento conceptual y otro perceptual:

la unidad -a veces inefable- de la metáfora, tiene como principio las cualidades ya aprehendidas en intelección campal, pero su línea principial puede ser muy distinta. Esta línea de intelección es justo la línea del 'hacia' en cuanto tal ${ }^{68}$.

${ }_{68}$ Zubiri. Inteligencia y razón, Alianza, Madrid, 1983, p. 155. 
Toda idea de Dios, pues, es máximamente inadecuada. Por su menor dimensión representativa, la afirmación de esta simple aprehensión no solo posee un grado de conformidad muy limitado, sino que es inadecuada, infinitamente inadecuada. Que Dios sea vida es una afirmación que no es independiente de la representación judicativa de la propia vida -la que cada cual experimenta en primera persona-, y aun de la de los demás seres biológicos. Lo que es tanto como decir que este sentido de la vida vegetal, animal y personal está ínsito en la afirmación según la cual "Dios es vida". Por supuesto, Dios no es vida en el mismo sentido de las realidades vivas no personales. Esto hay que negarlo, para afirmar que Dios es vida en otro sentido. La significación de la vida divina es otra que la de la vida de los seres vivos que no son personas como nosotros. Y respecto de las vidas personales, habría que determinar si la vida de Dios es vida en otro sentido que las vidas personales finitas, o si es vida en el mismo sentido que ellas, pero no en el mismo grado. En este otro sentido, cabría afirmar solamente por eminencia que Dios sea vida. Tal eminencia es una afirmación analógica depurada por la negación: es la infinita direccionalidad mentada por la vitalidad divina.

\section{Perspectivas}

Según Zubiri, en conclusión, la vida intramundana tiene sus estratos, los que de buenas a primeras no se ve cómo pudieran servir para entender a Dios, cuya vida posee un momento radicalmente extramundano. Pero, lo que sí parece útil para comprender a Dios es que la vida también es caracterizable por su modo de ser o realidad: la de una autoposesión que en el caso de las personas alcanza un despliegue superlativo. Zubiri piensa que la noción de autoposesión sirve para representar -hasta donde ello tenga sentido, esto es, de manera conforme pero a la vez inadecuada- a la realidad absolutamente absoluta de Dios, la que entonces sería afirmada como una realidad que se autoposee y es suya infinitamente: una suidad infinita. Por supuesto, no hay que perder de vista las acusadas diferencias tanto entre la vía de la analogía y la de la univocidad, como las divergencias que incluso se advierten entre la analogía tomista y la de Zubiri. Mientras la tomista es analogia entis y conduce a Dios en tanto en cuanto ente automoviente supremo, la analogía zubiriana, que es analogía tanto de la vida como de la realidad absolutas, una y otra apoyadas a radice en una aprehensión primordial de lo real, lleva a Dios en tanto en cuanto realidad-fundamento y vida plenaria. Y, contra 
las rutas abiertas por Escoto y Ockham, hoy proseguidas por parte del teísmo analítico, en la filosofía de Zubiri se halla sugerida una teoría analógica y metafórica de los enunciados teístas que echa mano de la convergencia en el lenguaje religioso de elementos perceptivos, ficticios y conceptuales, todos los cuales se recortan sobre el fundamento de un inamisible poder de la realidad ya dado primordialmente.

Sin embargo, varias son las cuestiones que habría que meditar más detalladamente. De ellas, para terminar, conviene destacar cuando menos las que siguen:

a) Desde ya, habría que pensar si la vida divina se afirma como más afín desde la primera o la tercera persona. En este sentido, contra la interpretación aquí sugerida, podría contraargumentarse que, dado que la vida más afín es la nuestra, entonces la noción de autoposesión habría de ser obtenida desde la precisa perspectiva de primera persona, la de la vida personal tal como la vive cada hombre en tanto que imagen y semejanza de la vida de Dios, y no desde la perspectiva de tercera persona, según la cual se idea y afirma por eminencia la vitalidad divina también -aunque no solo- a partir de la vida animal y vegetal. Así, la vida divina, en Zubiri, sería más afín a la de primera persona, no a la de tercera, dado que Dios es realidad-fundamento, nunca realidad-objeto.

Además, habría que añadir que la analógica afirmación religiosa puede ser parte no solo del logos, del que hemos hablado hasta aquí, sino también de la razón, que es otra modalidad intelectiva, una para la cual la vida de Dios no solo es una idea y una afirmación por analogía forjadas en el campo de lo real, sino que es un sistema de referencia para una búsqueda racional de sus rasgos profundos en el mundo y aun más allá de él. Entonces, la vitalidad divina ya no sería pura afirmación analógica de la realidad campal de Dios, sino que podría ser esbozo (todo lo inadecuado que se quiera) de su realidad esencial. Es un asunto que habría que precisar mejor, por cierto, pues no toda conceptuación pertenece al logos. Así como el concepto de átomo parece ser un concepto mediado por una elaboración racional, lo mismo parece ocurrir con muchas ideas sobre Dios. Lo que es tanto como decir que con el concepto del Dios vivo, o de la Vida que es plenaria y simplemente Dios, se hacen afirmaciones campales y razonamientos mundanales y también transmundanales ${ }^{69}$.

69 Para una interpretación acerca de los contornos de la razón religiosa según Zubiri, cf. E. Solari, op. cit., pp. 300ss. 
También, a la via eminentiae (según la cual la afirmación analógica de la vida de Dios está depurada por la negación y apunta en dirección infinita) podría oponerse una matización de envergadura. Pues lo que pretende Zubiri no es postular una vida divina a fuerza de negar caracteres de la humana y las demás. El punto es que habría una absolutidad de la fundamentalidad dada de igual modo que nuestra absolutidad (relativa). Y tal sería el fundamento de la analogía. Dios no es una pura idea inventada a fuerza de negaciones de lo dado: eso es lo que cree Feuerbach, mas no Zubiri.

b) Por otra parte, cabría apelar todavía no solo a una teoría del lenguaje religioso, como la tomista, la univocista y la que cabe entresacar del propio Zubiri, sino a una filosofía fenomenológica de la vida y del cristianismo tan conspicua como la de Michel Henry. La suya es una filosofía que no se atrinchera en el lenguaje, sino que piensa que la fenomenología puede retroceder tan originariamente que termine por manifestar unos fenómenos arcaicos, no lingüísticos ni siquiera ontológicos. Y es que, según Henry, más allá (o, mejor, más acá) del ser y el lenguaje está ya la vida. De la vida, dice, la biología no tiene ninguna idea. La vida no se manifiesta en el horizonte del mundo ni en el lenguaje del ser. Su verdad no es mundanal, pues entre el mundo y la vida, y especialmente entre el mundo y el cristianismo, hay una mutua exclusión, una irreductibilidad esencial. En palabras de Henry: "Vida designa una manifestación pura, irreductible a la del mundo no obstante, una revelación original que no es la revelación de otra cosa y que no depende de nada distinto, sino una revelación de sí"70. La vida, pues, no aparece ni se muestra mundanalmente sino como mera significación noemática irreal o vacía. Mas, en su realidad primigenia ella se manifiesta de otra manera, precisamente como sensibilidad, afectividad, como pathos:

vemos a los seres vivos pero nunca vemos su vida [...] Esta Vida fenomenológica que se experimenta a sí misma, esta vida real que es la nuestra, que habita cada una de nuestras alegrías y sufrimientos, de nuestros deseos y temores y, antes que nada, la más humilde de nuestras sensaciones, es sin embargo la gran ausente de la tradición filosófica y cultural a la que pertenecemos ${ }^{71}$.

70 Cf. Michel Henry, Yo soy la verdad. Para una filosofía del cristianismo, trad. J. Teira, Sígueme, Salamanca, 2001, p. 44.

71 M. Henry, Yo soy..., pp. 51 y 53; vid. p. 62. 
Dios es Vida para el cristianismo, agrega Henry, pero en un sentido que conviene aclarar. Si para la filosofía griega el ser humano es por su

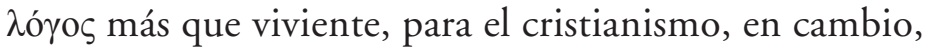

la Vida es más que el hombre [...] La vida, que no dice palabra, lo sabe todo, en todo caso mucho más que la razón. Y ello en el hombre tanto como en Dios [...] La Vida es más que el viviente ${ }^{72}$.

No hay que perder de vista que la de Zubiri es una fenomenología cuyo punto de anclaje no es -como la de Ortega- la vida. La suya es una fenomenología de la realidad y de su actualidad en la intelección. Sin embargo, Zubiri podría decir con Henry que Dios no es un viviente más, una realidad viva entre otras, sino la Vida plenaria misma: la realidad absolutamente absoluta, personal e inteligente. Y también, podría estar de acuerdo con Henry en que una teoría del lenguaje religioso y de los predicados teológicos, como el de vida, requiere una meditación fenomenológica que desborda el ámbito del lenguaje, de la consciencia intencional y aun de la compresión del ser.

c) Fenomenologías como las de Zubiri y Henry insisten en que la Vida, el Dios vivo, es inseparable del judeocristianismo. Pero, es posible por cierto seguir prolongando y afinando estas vías fenomenológicas. La filosofía de Henry conoce seguidores y polémicas ${ }^{73}$. Y lo propio cabe decir de la filosofía de Zubiri. Si para este, que el Dios de Israel y de Jesús sea viviente y personal significa que es una eminente autoposesión, para un discípulo de Zubiri, Antonio González, la vida personal de Dios puede ser mejor y más detalladamente comprendida (hasta donde esto es posible) diciendo que toda vida personal es no solo ni siempre una autoposesión, sino más bien una suerte de apertura, distensión o liberación. La persona viva es tal no tanto porque en algunos de sus actos se autoposee, sino por cuanto precisamente a través de sus actos $-\mathrm{y}$ justo porque en los mismos se hace presente una alteridad radical, la de las cosas actualizadas en ellos- ya ha acontecido una liberación autonomi-

M. Henry, Yo soy..., p. 63 (vid. p. 64).

73 Vid. por ej. T. Dika y W. Hackett, "La phénoménologie et le concept de vie. Un entretien avec Renaud Barbaras", en Revue de la philosophie française et de langue française Vol. $19 \mathrm{~N}^{\circ} 2$, 2011, pp. 153-179, esp. pp. 177-178, todo lo cual es explanado con detalle en R. Barbaras, Introduction à une phénoménologie de la vie, Vrin, París, 2008. 
zadora del encadenamiento estimúlico ${ }^{74}$. Recientemente, González ha dado un paso más. Los actos, dice ahora, son el surgir de las cosas. Son, por lo mismo, nuestra más íntima textura,

lo más cercano a nosotros mismos. Los actos constituyen lo que usualmente se llama 'vida' (no en un sentido biológico), o 'praxis' (no en un sentido pragmático), o 'existencia' (no en un sentido existencialista) $)^{75}$.

Y no solamente esto. González parece llevar a término la afirmación zubiriana del curso PD que ya citamos, y según la cual "la acción vital divina es inemergente, es puramente subsistente". Esta inemergencia es, en los términos de González, una 'insurgencia'. Mientras el ser humano es una vida tramada de actos abiertos, distensos y por ende personales, actos de diferente tipo que se entrelazan de muy diversas maneras, pero en todos los cuales aparecen, surgen cosas, Dios tampoco es una cosa que surja o aparezca, sino actualidad por excelencia, el puro surgir, la génesis de todas las cosas, el 'in-surgente'. En tanto que tal, Dios es un infinito surgir, y lo es porque no está limitado por cosa alguna que pueda surgir, ya que de Él surgen todas las cosas:

un surgir infinito, un surgir ilimitado, un surgir insurgente, es un surgir que no culmina en cosa. $\mathrm{Y}$, al no culminar en cosa, el surgir no se distingue del surgir. El surgir infinito es un surgir 'uno'. La pluralidad de surgires requiere la pluralidad de representaciones, y la pluralidad de representaciones abandona el ámbito del puro surgir, y se traslada a las cosas: es la idolatría. Frente a la idolatría, el puro surgir es un surgir vivo. En cuanto un surgir 'uno', separado de todas las cosas, podemos atribuirle la bondad infinita, a diferencia de la bondad limitada de los actos finitos en su unidad con lo que surge. En cuanto surgir vivo, le corresponde la belleza originaria e infinita, propia de la vida ${ }^{76}$.

Así, pues, también conviene explorar si y cómo la vida de Dios puede ser la de un actus purus -trastrocando, claro, la terminología aristotélica.

74 A. González, Estructuras de la praxis. Ensayo de filosofía primera, Trotta, Madrid, 1997, pp. 93-94; y Teología de la praxis evangélica. Ensayo de teología fundamental, Sal Terrae, Santander, 1999, pp. 87-88.

75 A. González, "La génesis de todas las cosas", en Cuadernos salmantinos de filosofía Vol. 40, 2013, p. 549.

76 A. González, "La génesis...", p. 555. 